\title{
Fraturas ipsilaterais de fêmur e pelve (quadril flutuante): análise prospectiva de 16 casos
}

\author{
Management of ipsilateral fractures of the femur and \\ pelvis (floating hip): a prospective study on 16 cases
}

\author{
Vincenzo Giordano', Ney Pecegueiro do Amaral ${ }^{2}$, Henrique Rios ${ }^{3}$, \\ Carlos Eduardo Franklin ${ }^{4}$, AleXandre Pallottino ${ }^{4}$
}

\section{RESUMO}

Objetivo: Analisar 16 casos de fraturas ipsilaterais de fêmur e pelve, descrevendo lesões associadas, tratamento, resultados e complicações. Métodos: Foram estudados 16 pacientes (13 do sexo masculino e três do feminino, com idade média de 27,8 anos [variando de 19,1 a 35,6 anos]), apresentando fratura ipsilateral de fêmur e pelve ("quadril flutuante"). $O$ mecanismo de lesão envolveu acidente em via pública em todos os pacientes: colisão auto-auto em 10, colisão auto-objeto estático em três e atropelamento em três. Todos os pacientes apresentaram lesões associadas. De acordo com a classificação de Liebergall $e$ t al, oito pacientes eram do tipo A, seis do tipo $B$ e dois pacientes do tipo $\mathrm{C}$. O momento da cirurgia foi determinado pelo status hemodinâmico do paciente e sua resposta às manobras de ressuscitação inicial e pelas condições de partes moles nos locais de lesão. As lesões foram fixadas definitivamente em média no 12 ${ }^{\circ}$ dia após o acidente (variando de

* Trabalho realizado no Serviço de Ortopedia e Traumatologia do Hospital Municipal Miguel Couto - HMMC - Rio de Janeiro (RJ), Brasil.

1. Médico Assistente e Coordenador do Programa de Residência Médica do Serviço de Ortopedia e Traumatologia do Hospital Municipal Miguel Couto - HMMC - Rio de Janeiro (RJ), Brasil.

2. Chefe do Serviço de Ortopedia e Traumatologia do Hospital Municipal Miguel Couto - HMMC - Rio de Janeiro (RJ), Brasil.

3. Residente Serviço de Ortopedia e Traumatologia do Hospital Municipal Miguel Couto - HMMC - Rio de Janeiro (RJ), Brasil.

4. Assistente do Serviço de Ortopedia e Traumatologia do Hospital Municipal Miguel Couto - HMMC - Rio de Janeiro (RJ), Brasil.

Endereço para correspondência: Dr. Vincenzo Giordano, Rua Aristides Espínola, 11/301, Leblon - 22440-050 - Rio de Janeiro, RJ. Tel./fax: (21) 22746830. E-mail: sot.hmmc@terra.com.br

Recebido em 30/11/06. Aprovado para publicação em 14/8/07.

Copyright RBO2007 nove a 19 dias). Os procedimentos foram realizados em único tempo operatório. $O$ período médio de internação foi de 28 dias (variando de 16 a 120 dias). Nenhum paciente abandonou o tratamento e o seguimento médio foi de 26 meses (variando de 20 a 51 meses). Os resultados foram avaliados segundo os critérios de d'Aubigné e Postel modificados por Matta et al. Resultados: Nenhum paciente evoluiu a óbito. Utilizando os critérios de d'Aubigné e Postel modificados, os resultados foram excelentes em três casos, bons em nove, regulares em três e ruim em um. Os resultados regulares deveram-se principalmente à existência de dor à deambulação, tornando-a limitada, mesmo com o uso de muletas. Um paciente apresentou resultado final ruim após ter sofrido fratura ipsilateral aberta de tíbia, aberta de fêmur e de rebordo posterior com luxação de quadril. Considerações finais: Fraturas ipsilaterais de fêmur e pelve são lesões complexas e que requerem treinamento específico para seu manejo. Resultados satisfatórios podem ser esperados quando essas lesões são tratadas por profissionais familiarizados com a anatomia da região do anel pélvico. A existência de envolvimento do acetábulo piora o prognóstico do paciente.

Descritores - Fraturas do fêmur/cirurgia; Ossos pélvicos/lesões; Ace-
tábulo; Lesões do quadril

\section{ABSTRACT}

Objective: Review 16 cases of ipsilateral femur and pelvis fractures, describe associated lesions, treatment, results, and complications. Methods: 16 patients were studied (13 male and three female, mean age of 27.8 years [ranging from 19.1 to 35.6 years]), with ipsilateral fractures of the femur and pelvis (floating hip). The lesion mechanism involved a street 
accident for all patients: cart-to-car collision in 10 cases, car-to-object collision in 3 cases, and running over in three cases. All patients presented with associated lesions. According to Liebergall et al classification, eight patients were type $A$, six were type $B$, and two were type $C$. The time of surgery was determined by the hemodynamic status of the patient and patient response to initial resuscitation maneuvers, and by soft part condition at the sites of lesion. On the average, lesions were definitely fixated on the $12^{\text {th }}$ day after the accident (ranging from 9 to 19 days). Procedures were performed in a single surgery. Mean hospitalization time was 28 days (ranging from 16 to 120 days). No patient abandoned the treatment and mean follow-up was of 26 months (ranging from 20 to 51 months). Results were evaluated according to the criteria of d'Aubigné and Postel modified by Matta et al. Results: None of the patients died. Using the modified d'Aubigné and Postel criteria, results were excellent in three cases, good in nine, regular in three, and poor in one. Regular results were mostly due to the presence of pain on walking, main walking limited even with the use of crutches. One patient evolved to a poor final result after an ipsilateral open fracture of the tibia, open fracture of the femur and of the posterior border with hip displacement. Final Comments: Ipsilateral fractures of the femur and pelvis are complex lesions requiring specific management training. Satisfactory results can be expected when these lesions are treated by professionals who are acquainted with the anatomy of the pelvic ring anatomy. Acetabulum involvement makes for a worse prognosis for the patient.

Keywords - Femoral fractures/surgery; Pelvic bones/injuries; Acetabulum; Hip injuries

\section{INTRODUÇÃO}

Fraturas ipsilaterais de estruturas adjacentes são relativamente comuns em pacientes vítimas de múltiplo trauma ${ }^{(1)}$. Na maioria dos casos, ocorre em decorrência da violência do tráfego, como resultado de acidentes automobilísticos ${ }^{(2-5)}$. Tradicionalmente, essas lesões têm sido reconhecidas pelo termo "flutuante", embora alguns autores venham recentemente questionando tal denominação por acharem-na vaga e imprópria( ${ }^{(6)}$.

O termo "quadril flutuante" vem sendo empregado para descrever fraturas ipsilaterais de fêmur e pelve. Três diferentes padrões de lesão foram descritos, refletindo, principalmente, a energia cinética necessária para provocar as fraturas e o potencial de perda sanguínea existente ${ }^{(7)}$. O tipo A corresponde à fratura ipsilateral de fêmur e acetábulo; o tipo B, à fratura ipsilateral de fêmur e anel pélvico; e o tipo C, à fratura ipsilateral de fêmur, acetábulo e anel pélvico. Apesar de tratar-se de afecção traumática incomum, há elevada taxa de morbidade e de mortalidade, sendo relativamente freqüente o surgimento de complicações nesse padrão específico de associação( ${ }^{(7-8)}$.

Seu manejo é extremamente difícil, tanto na fase aguda quanto na fase tardia ${ }^{(8-9)}$. Recentemente, com a introdução do conceito de "controle do dano", fixação temporária das lesões existentes é medida salvadora de vida, fazendo parte das manobras de ressuscitação inicial como adjuvante ao controle de hemorragias ${ }^{(10)}$. Diversas opções vêm sendo descritas para a

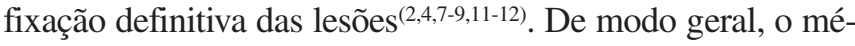
todo de tratamento depende do padrão e da localização da fratura e das condições de partes moles ${ }^{(8)}$.

No presente estudo, foram analisados 16 casos de fraturas ipsilaterais de fêmur e pelve, descrevendo lesões associadas, tratamento, resultados e complicações.

\section{MÉTODOS}

No período de janeiro de 2000 a janeiro de 2005, 16 pacientes (13 do sexo masculino e três do feminino, média de idade de 27,8 anos [variando de 19,1 a 35,6 anos]) foram admitidos em nossa instituição pelo Setor de Emergência apresentando fratura ipsilateral de fêmur e pelve ("quadril flutuante"). O mecanismo de lesão envolveu acidente em via pública em todos os casos: colisão auto-auto em 10, colisão auto-objeto estático em três e atropelamento em três. Todos os pacientes apresentaram lesões associadas, com média de Escore de Gravidade de Lesão (Injury Severity Score) de 21 (variando de 15 a 23). Entre os pacientes, três sofreram traumatismo craniencefálico (TCE) leve, com Glasgow Coma Scale (Escala de Coma de Glasgow) superior a 8 , não necessitando de ventilação mecânica; seis tiveram contusão pulmonar, dois deles requerendo drenagem do tórax na fase aguda e um, 24 horas após a admissão hospitalar. Todos permaneceram em Unidade Intermediária do Serviço de Cirurgia Geral da instituição em média por uma semana (variando de cinco a 11 dias). Lesão de vísceras abdominais foram detectadas em dois pacientes, ambos submetidos à laparotomia exploratória de urgência; um deles teve rotura de baço e foi esplenectomizado na fase aguda e o outro, lesão hepática parenquimatosa, sendo rafiado. Em 10 pacientes ocorreram lesões músculoesqueléticas associadas; quatro sofreram luxação traumática posterior do quadril ipsilateral (associada à fratura do acetábulo), todas reduzidas de forma fechada nas primeiras seis horas de lesão e colocados os pacientes em tração esqueléti- 
ca; três sofreram fratura ipsilateral de tíbia, todas abertas, sendo submetidos a desbridamento, limpeza cirúrgica e fixação externa imediatamente após liberação para o centro cirúrgico; dois sofreram fraturas fechadas nos membros inferiores, sendo uma da diáfise umeral e outra do terço distal dos ossos do antebraço; ambas foram tratadas cirurgicamente durante a estabilização das lesões no membro inferior; e um paciente teve fratura ipsilateral do corpo da escápula e contralateral diafisária do fêmur, fechada. A fratura da escápula foi manejada conservadoramente e a do fêmur foi estabilizada na emergência com fixador externo, convertido posteriormente para haste intramedular bloqueada retrógrada, durante a fixação do "quadril flutuante". Os principais dados dos pacientes são apresentados na tabela 1 .

TABELA 1

Dados demográficos dos pacientes

\begin{tabular}{lc}
\hline & Pacientes (n = 16) \\
\hline Idade (anos) [variação] & $27,8[19,1-35,6]$ \\
Sexo (masculino/feminino) & $13 / 3$ \\
Lado (esquerdo/direito) & $11 / 5$ \\
Mecanismo de trauma & \\
$\checkmark$ colisão auto-auto & 10 \\
$\checkmark$ colisão auto-objeto estático & 3 \\
$\checkmark$ atropelamento & 3 \\
Lesões associadas & \\
$\checkmark$ TCE & 3 \\
$\checkmark$ contusão pulmonar & 6 \\
$\checkmark$ lesão de víscera abdominal & 2 \\
$\checkmark$ outras lesões músculo-esqueléticas & 10 \\
\hline
\end{tabular}

Fonte: SOT-HMMC, 2006

De acordo com a classificação de Liebergall et al, oito pacientes eram do tipo $\mathrm{A}$, seis do tipo $\mathrm{B}$ e dois do tipo $\mathrm{C}^{(7)}$. A fratura de fêmur ocorreu na região diafisária em 14 pacientes, na região intertrocanteriana em um e segmentar de colo e diáfise em um. Das 10 fraturas do acetábulo, cinco eram do rebordo posterior, uma de rebordo e coluna posterior, duas transversas com rebordo posterior, uma de coluna anterior e uma da dupla coluna ${ }^{(13)}$. Das oito lesões do anel pélvico, uma era estável, três eram rotacionalmente instáveis e quatro, verticalmente instáveis ${ }^{(14)}$. Entre os pacientes, cinco apresentaram fratura aberta do fêmur e um da pelve. As fraturas abertas do fêmur foram classificadas de acordo com Gustilo et al, sendo: uma grau 1 , duas grau 2 e duas grau $3 \mathrm{~A}^{(15-16)}$. A fratura aberta da pelve foi classificada de acordo com Jones et al, sendo classe $1^{(17)}$.
O momento da cirurgia foi determinado pelo status hemodinâmico do paciente e sua resposta às manobras de ressuscitação inicial e pelas condições de partes moles nos locais de lesão. Os cinco pacientes com fratura aberta diafisária de fêmur foram submetidos a desbridamento, limpeza cirúrgica e fixação externa imediatamente após liberação para o centro cirúrgico. Outros sete pacientes com fratura diafisária de fêmur tiveram estabilização inicial da lesão com fixador externo e dois foram colocados em tração esquelética transtibial. $\mathrm{O}$ paciente com fratura intertrocanteriana e o com fratura segmentar de colo e diáfise femorais permaneceram em repouso no leito hospitalar, fora de tração; quatro pacientes com luxação traumática posterior do quadril e fratura do acetábulo foram submetidos à redução fechada da luxação e instalação de tração esquelética transtibial, conforme mencionado anteriormente; e cinco outros pacientes com fratura de acetábulo foram colocados em tração esquelética transtibial e um aguardou cirurgia em repouso no leito hospitalar. O paciente com fratura aberta da asa do ilíaco foi submetido a desbridamento e limpeza cirúrgica imediatamente após liberação para o centro cirúrgico. Os demais pacientes com lesão do anel pélvico receberam dispositivos de fixação externa e, nos quatro com instabilidade vertical, tração esquelética transtibial. Os principais dados das lesões são apresentados na tabela 2.

TABELA 2

Dados das lesões

Pacientes $(n=16)$

Classificação de Liebergall et $a^{(7)}$

$\checkmark$ Tipo A

8

$\checkmark$ Tipo B

$\checkmark$ Tipo $C$

2

Fratura de fêmur

$\checkmark$ diafisária

$\checkmark$ intertrocanteriana

$\checkmark$ colo + diáfise

$\checkmark$ aberta

14

1

Fratura de acetábulo

$\checkmark$ rebordo posterior $\quad 5$

$\checkmark$ coluna anterior

$\checkmark$ rebordo + coluna posterior

$\checkmark$ transversa + rebordo posterior

$\checkmark$ dupla coluna

1

1

2

1

Lesão do anel pélvico

$\checkmark$ estáveis

$\checkmark$ rotacionalmente instáveis

$\checkmark$ verticalmente instáveis

$\checkmark$ aberta

1

3

4

1

Fonte: SOT-HMMC, 2006 
As lesões foram fixadas definitivamente em média no $12^{\circ}$ dia após o acidente (variando de nove a 19 dias). Os procedimentos foram realizados em único tempo operatório: nove fraturas diafisárias de fêmur foram tratadas com haste intramedular bloqueada (HIB) anterógrada, cinco com placa de compressão dinâmica $\left(D C P^{\circledR}\right)$ de grandes fragmentos e uma com parafuso condiliano dinâmico $\left(D C S^{\circledR}\right)$, a fratura intertrocanteriana foi tratada com parafuso deslizante do quadril $\left(D H S^{\circledR}\right)$ de $135^{\circ}$ e a do colo femoral, com três parafusos canulados de grandes fragmentos. Todas as fraturas do acetábulo com desvio do componente posterior $(n=9)$ foram manejadas pelo acesso posterior de Kocher-Langenbeck; duas delas tiveram também abordagem anterior pelo acesso ilioinguinal e uma foi fixada de forma percutânea retrógrada com parafuso canulado de grandes fragmentos. Todas as fraturas instáveis da pelve tiveram o componente anterior fixado pelo acesso de Pfannenstiel e o posterior, pela primeira janela do acesso ilioinguinal $(n=3)$ ou percutaneamente com o uso de fixação transilíaca com placa $(\mathrm{n}=1)^{(18)}$. Os principais dados do tratamento são apresentados na tabela 3.

TABELA 3

Dados do tratamento dos pacientes

Pacientes $(n=16)$

\begin{tabular}{|c|c|}
\hline Fratura de fêmur & \\
\hline$\checkmark$ HIB anterógrada & 9 \\
\hline$\checkmark D C P^{\otimes}$ & 5 \\
\hline$\checkmark D H S^{\circledR}$ & 1 \\
\hline$\checkmark D C S^{\circledR}$ & 1 \\
\hline$\checkmark$ parafusos canulados & 1 \\
\hline Fratura de acetábulo & \\
\hline$\checkmark$ Kocher-Langenbeck & 9 \\
\hline$\checkmark$ ilioinguinal & 2 \\
\hline$\checkmark$ percutânea retrógrada & 1 \\
\hline Lesão do anel pélvico & \\
\hline$\checkmark$ Pfannenstiel & 7 \\
\hline$\checkmark$ ilioinguinal (1a janela) & 3 \\
\hline$\checkmark$ percutânea posterior & 1 \\
\hline
\end{tabular}

Fonte: SOT-HMMC, 2006

O período médio de internação foi de 28 dias (variando de 16 a 120 dias). Profilaxia antimicrobiana foi realizada por 24 horas em todos os pacientes com cefalosporinas de primeira geração. Nos pacientes com fratura aberta, adotamos protocolo padrão de antibioticoprofilaxia, associando-se aminoglicosídeo por 48 horas nas fraturas $3 \mathrm{~A}$ de Gustilo et al ${ }^{(15-16)}$. Profilaxia das complicações tromboembólicas foi aplicada de forma mecânica em todos os pacientes. Profilaxia para ossificação heterotópica foi prescrita em todos os pacientes submetidos ao acesso posterior de Kocher-Langenbeck, com indometacina $75 \mathrm{mg}$ ao dia durante seis semanas. Após a alta hospitalar, os pacientes foram seguidos em regime ambulatorial, com visitas na terceira, sexta e $12^{\mathrm{a}}$ semanas, sexto e $12^{\circ}$ meses e uma vez por ano após o primeiro ano de acompanhamento. Carga progressiva sobre o membro lesado foi permitida após três meses das cirurgias definitivas, inicialmente com auxílio de muletas e gradualmente sem proteção.

Nenhum paciente abandonou o tratamento e o seguimento médio foi de 26 meses (variando de 20 a 51 meses). Os resultados foram avaliados segundo os critérios de d'Aubigné e Postel modificados por Matta et $a l^{(19-20)}$.

\section{RESULTADOS}

Nenhum paciente evoluiu a óbito. Utilizando os critérios de d'Aubigné e Postel ${ }^{(19)}$ modificados, os resultados foram excelentes em três casos, bons em nove, regulares em três e ruim em um (figuras 1 e 2). Os resultados regulares deveramse principalmente à existência de dor à deambulação, tornando-a limitada mesmo com o uso de muletas. Todos sofreram fratura ipsilateral de fêmur e acetábulo (Liebergall et al tipo $\left.\mathrm{A}^{(7)}\right)$, sendo duas isoladas de rebordo posterior e uma de coluna e rebordo posterior. Em um paciente o resultado final foi ruim; ele havia sofrido fratura ipsilateral aberta de tíbia, aberta de fêmur e do rebordo posterior com luxação de quadril (Liebergall et al tipo $\left.\mathrm{A}^{(7)}\right)$. Fora manejado na Emergência com fixador externo de tíbia e fêmur e teve seu quadril reduzido de forma fechada. Foi posteriormente reoperado para retirada do fixador externo do fêmur e colocação de placa de compressão dinâmica e fixação do rebordo posterior do acetábulo. Evoluiu com infecção profunda na coxa operada e instabilidade no quadril ipsilateral. Foi submetido a desbridamentos seriados e evoluiu com pseudartrose da diáfise e necrose da cabeça femoral. Após cura do processo infeccioso, foi reoperado para nova osteossíntese com placa na diáfise do fêmur e realização de artroplastia total do quadril ipsilateral, cimentada. Após oito meses da lesão, o paciente teve as fratura de tíbia e fêmur consolidadas, mas perdeu completamente o arco de movimento do joelho. A prótese de quadril apresentava-se fixa na última revisão ambulatorial.

\section{DISCUSSÃO}

O manejo das fraturas ipsilaterais de fêmur e pelve apresenta inúmeras dificuldades. Em geral, os pacientes são politraumatizados e procedimentos definitivos de estabilização das 

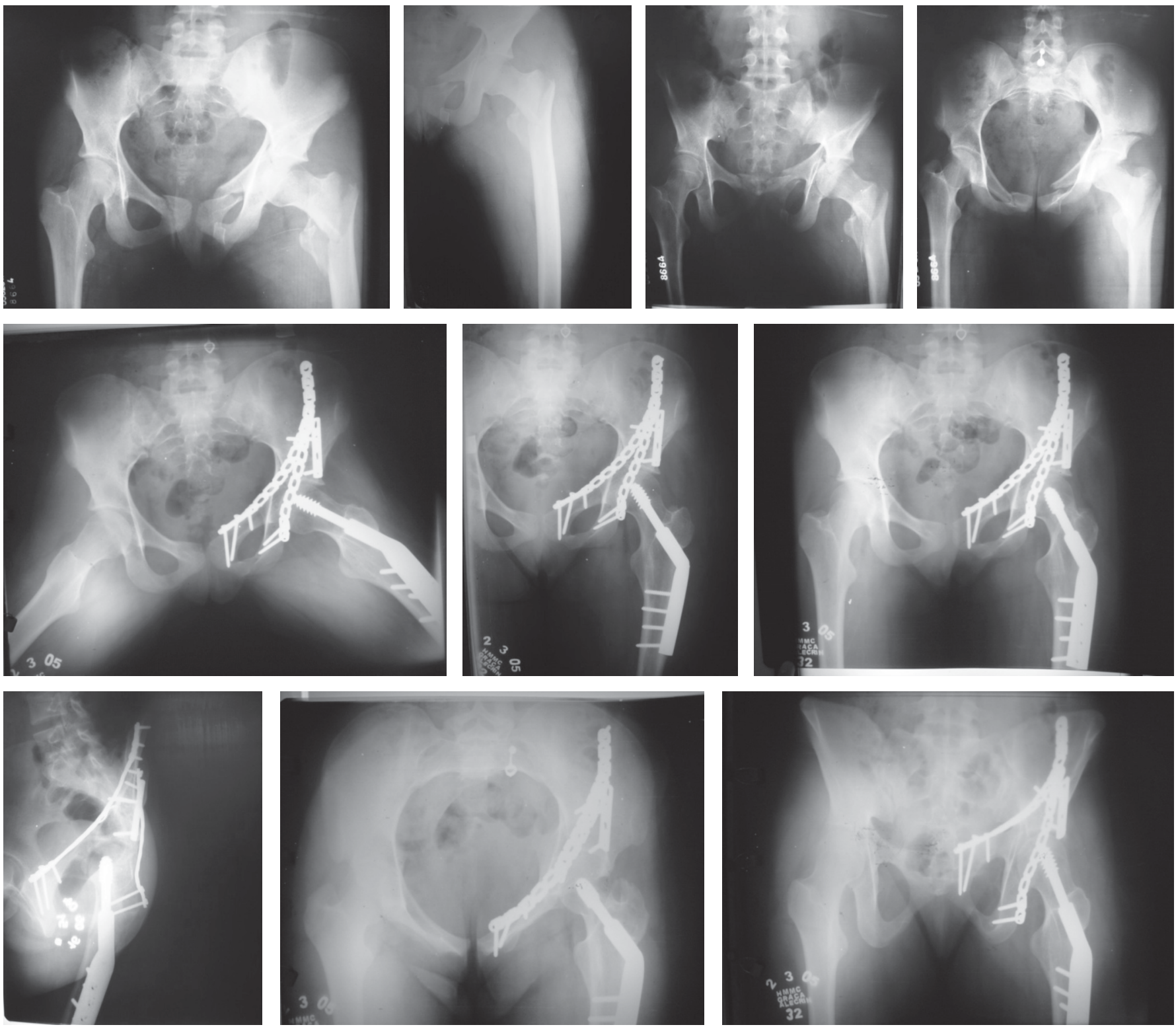

Figura 1 - Radiografias mostrando fratura ipsilateral intertrocanteriana e da dupla coluna do acetábulo (Liebergall tipo A) à esquerda. Paciente foi submetida à osteossíntese com $D H S^{\otimes}$ de $135^{\circ}$ e dupla abordagem para a fratura do acetábulo. Seguimento de 28 meses.

lesões músculo-esqueléticas devem ser adiados até que haja adequada recuperação do trauma inicial ${ }^{(10,21)}$. Abordagem multidisciplinar é fundamental na fase aguda de ressuscitação dos pacientes, uma vez que tanto a lesão do anel pélvico quanto a fratura do fêmur podem provocar instabilidade hemodinâmica $^{(10)}$. Nesse contexto, a utilização de dispositivos de fixação externa vem sendo demonstrada como medida útil e eficaz no manejo primário de fraturas femorais e pélvicas em pacientes com múltiplas lesões associadas ${ }^{(2,21)}$. Independente- mente do padrão e da gravidade das lesões ortopédicas, o objetivo do tratamento na fase de ressuscitação é obter equilíbrio hemodinâmico e impedir danos adicionais ao paciente.

Müller et al têm proposto que o tratamento definitivo do "quadril flutuante" deve seguir as recomendações específicas para cada uma das lesões existentes ${ }^{(8)}$. Fixação intramedular com haste bloqueada é atualmente o tratamento de escolha para a maioria das fraturas diafisárias de fêmur na população esqueleticamente madura $^{(23)}$. Diversos autores, manejando fra- 

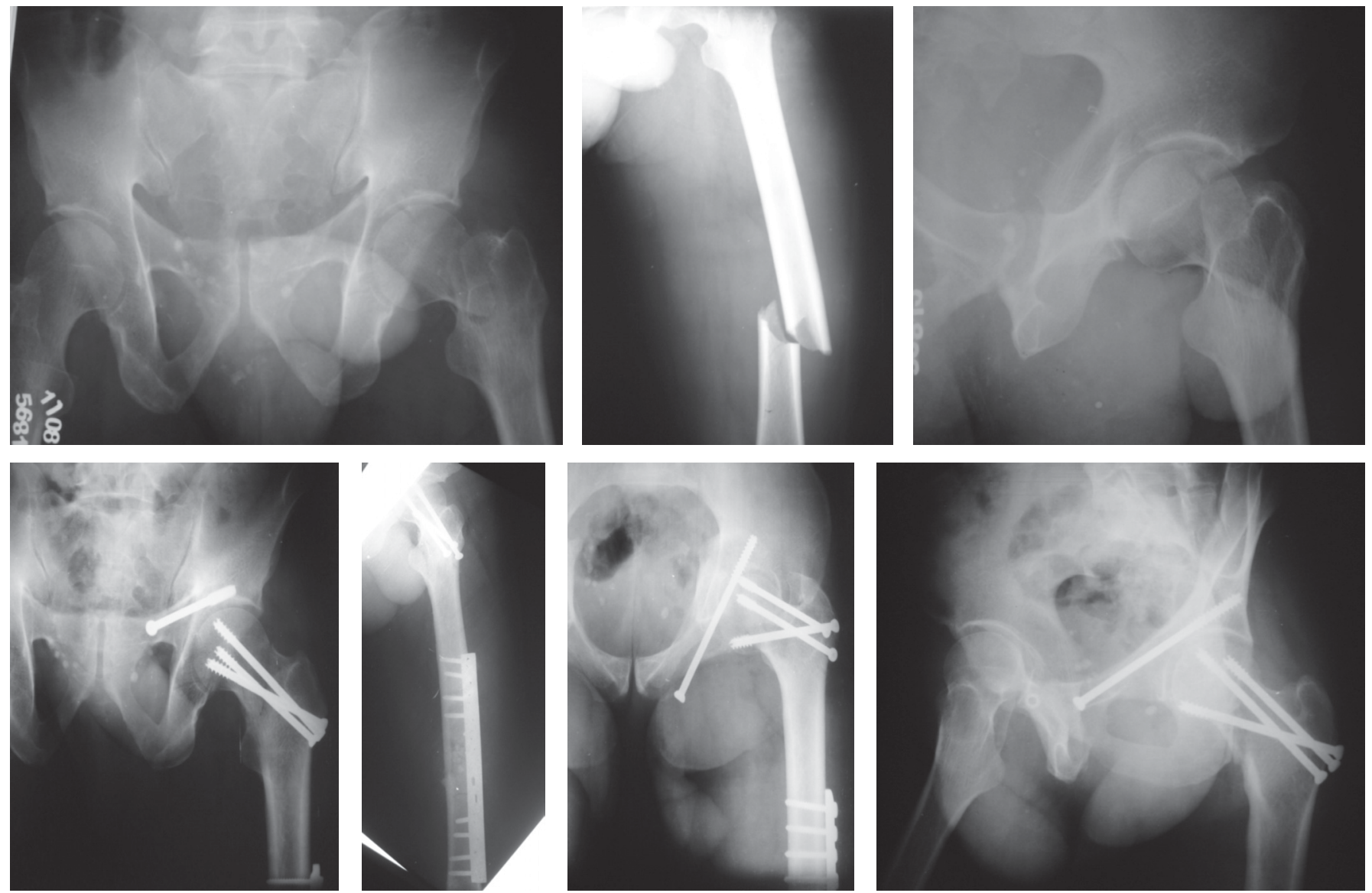

Figura 2 - Radiografias mostrando fratura ipsilateral diafisária proximal de fêmur (subtrocanteriana) e transversa com rebordo posterior do acetábulo (Liebergall tipo A) à esquerda. Paciente foi submetida à osteossíntese com $D C S^{\circledR}$ e abordagem posterior para a fratura do acetábulo. Seguimento de 23,5 meses.

turas ipsilaterais de fêmur e pelve, advogam seu uso na fratura femoral ${ }^{(7-9,24)}$. Algumas situações, no entanto, nos parecem mais apropriadas para o uso de outros dispositivos de fixação. Em nossa casuística, preferimos o uso de placa de compressão dinâmica quando o traço de fratura era simples e o canal medular menor do que $8 \mathrm{~mm}$. Nesses pacientes foram buscadas estabilidade absoluta e consolidação direta. Em três outros pacientes, com traços mais proximais no fêmur, optamos pelo uso do $D C S^{\circledR}$, do $D H S^{\circledR}$ e de parafusos canulados, respectivamente, em uma fratura subtrocantérica, uma intertrocantérica e uma de colo.

Fraturas do acetábulo são lesões de abordagem cirúrgi$\mathrm{ca}^{(13,20)}$. Em nossa experiência, encontramos 10 casos desse tipo de lesão. Em dois pacientes ocorreu fratura associada da pelve, além da fratura ipsilateral de fêmur, denotando trauma de alta energia. Assim como outros autores, observamos maior incidência de fratura do rebordo posterior do acetábulo(25-26).
Embora esse seja o padrão mais comumente encontrado e, portanto, de mais fácil manejo, nossos quatro resultados insatisfatórios (três regulares e um ruim) foram vistos em pacientes com fratura do rebordo posterior. Rommens et al apontam a dificuldade na reconstrução do rebordo posterior, osteonecrose parcial e complicações relacionadas ao acesso de Kocher-Langenbeck como os principais fatores relacionados aos resultados insatisfatórios ${ }^{(27)}$. Esses autores relatam que, mesmo em mãos experientes, bons e excelentes resultados não excedem a $75 \%$. Adequado planejamento pré-operatório e cuidadosa manipulação das partes moles têm sido citados como formas de reduzir o número de complicações do manejo cirúrgico das fraturas do acetábulo( ${ }^{(8)}$.

Lesões pélvicas devem ser tratadas com o objetivo de restaurar de forma anatômica o formato do anel ósseo. Parece bem estabelecido na literatura que lesões comprometendo a parte posterior do anel pélvico, determinando instabilidade 
vertical, devem ser tratadas com fixação posterior ${ }^{(28)}$. No SOTHMMC, quando há luxação sacroilíaca pura, preferimos o uso de abordagem anterior a essa articulação, com colocação de duas placas ortogonais. Quando a lesão é do tipo crescente, preferencialmente realizamos abordagem posterior sobre o sítio de fratura do ilíaco para redução anatômica e fixação com placa de reconstrução ${ }^{(29)}$. Utilizamos nesses casos uma placa suplementar para fixação transilíaca ${ }^{(18)}$. Nas lesões rotacionalmente instáveis, realizamos acesso anterior à sínfise púbica, com colocação de uma placa superiormente a essa estrutura.

\section{CONSIDERAÇÕES FINAIS}

Fraturas ipsilaterais de fêmur e pelve são lesões complexas e que requerem treinamento específico para seu manejo. Resultados satisfatórios podem ser esperados quando essas lesões são tratadas por profissionais familiarizados com a anatomia da região do anel pélvico. A existência de envolvimento do acetábulo piora o prognóstico do paciente.

\section{REFERÊNCIAS}

1. Khan MR, Mirdad TM. Musculoskeletal injuries in a major road accident. Ann Saudi Med. 1997;17(4):444-6.

2. Brainard BJ, Slauterbeck J, Benjamin JB. Fracture patterns and mechanisms in pedestrian motor-vehicle trauma: the ipsilateral dyad. J Orthop Trauma. 1992;6(3):279-82.

3. Hee HT, Wong HP, Low YP, Myers L. Predictors of outcome of floating knee injuries in adults: 89 patients followed for 2-12 years. Acta Orthop Scand. 2001;72(4):385-94.

4. Riemer BL, Butterfield SL, Burke CJ 3rd, Mathews D. Immediate plate fixation of highly comminuted femoral diaphyseal fractures in blunt polytrauma patients. Orthopedics. 1992;15(8):907-16.

5. Simpson NS, Jupiter JB. Complex fracture patterns of the upper extremity. Clin Orthop Relat Res. 1995;(318):43-53.

6. Agarwal A, Chadha M. Floating injuries: a review of the literature and proposal for a universal classification. Acta Orthop Belg. 2004;70(6):50914.

7. Liebergall M, Lowe J, Whitelaw GP, Wetzler MJ, Segal D. The floating hip. Ipsilateral pelvic and femoral fractures. J Bone Joint Surg Br. 1992; 74(1):93-100

8. Müller EJ, Siebenrock K, Ekkernkamp A, Ganz R, Muhr G. Ipsilateral fractures of the pelvis and the femur - floating hip? A retrospective analysis of 42 cases. Arch Orthop Trauma Surg. 1999;119(3-4):179-82.

9. Iotov A, Tzachev N, Enchev D, Baltov A. Operative treatment of the floating hip. J Bone Joint Surg Br. 2006;88-B(Suppl 1):160.

10. Roberts CS, Pape HC, Jones AL, Malkani AL, Rodriguez JL, Giannoudis PV. Damage control orthopaedics: evolving concepts in the treatment of patients who have sustained orthopaedic trauma. Instr Course Lect. 2005;54:447-62.
11. Browne RS, Mullan GB. Intertrochanteric fracture of the femur with ipsilateral central fracture of the acetabulum. Injury. 1980;11(3):251-3.

12. Lyddon DW Jr, Hartman JT. Traumatic dislocation of the hip with ipsilateral femoral fractures. A case report. J Bone Joint Surg Am. 1971; 53(5):1012-6.

13. Judet R, Judet J, Letournel E. Fractures of the acetabulum: classification and surgical approaches for open reduction. Preliminary report. J Bone Joint Surg Am. 1964;46:1615-46.

14. Tile M. Pelvic ring fractures: should they be fixed? J Bone Joint Surg Br. 1988;70(1):1-12. Review.

15. Gustilo RB, Anderson JT. Prevention of infection in the treatment of one thousand and twenty-five open fractures of long bones: retrospective and prospective analyses. J Bone Joint Surg Am. 1976;58(4):453-8.

16. Gustilo RB, Mendoza RM, Williams DN. Problems in the management of type III (severe) open fractures: a new classification of type III open fractures. J Trauma. 1984;24(8):742-6.

17. Jones AL, Powell JN, Kellam JF, McCormack RG, Dust W, Wimmer P. Open pelvic fractures. A multicenter retrospective analysis. Orthop Clin North Am. 1997;28(3):345-50.

18. Albert MJ, Miller ME, MacNaughton M, Hutton WC. Posterior pelvic fixation using a transiliac $4.5-\mathrm{mm}$ reconstruction plate: a clinical and biomechanical study. J Orthop Trauma. 1993;7(3):226-32.

19. d'Aubigné RM, Postel M. Functional results of hip arthroplasty with acrylic prosthesis. J Bone Joint Surg Am. 1954;36-A(3):451-75.

20. Matta JM, Anderson LM, Epstein HC, Hendricks P. Fractures of the acetabulum. A retrospective analysis. Clin Orthop Relat Res. 1986;(205): 230-40.

21. Scalea TM, Boswell SA, Scott JD, Mitchell KA, Kramer ME, Pollak AN. External fixation as a bridge to intramedullary nailing for patients with multiple injuries and with femur fractures: damage control orthopedics. J Trauma. 2000;48(4):613-21; discussion 621-3.

22. Giannoudis PV, Veysi VT, Pape HC, Krettek C, Smith MR. When should we operate on major fractures in patients with severe head injuries? Am J Surg. 2002;183(3):261-7.

23. Canadian Orthopaedic Trauma Society. Nonunion following intramedullary nailing of the femur with and without reaming. Results of a multicenter randomized clinical trial. J Bone Joint Surg Am. 2003;85A(11):2093-6.

24. Bonnevialle P, Blazy O, Conte P, Determe P, Combes JM, Mansat M. A critical study of a series of 30 floating hips. J Bone Joint Surg Br. 1997;79 (Suppl 1):28.

25. Alonso JE, Volgas DA, Giordano V, Stannard JP. A review of the treatment of hip dislocations associated with acetabular fractures. Clin Orthop Relat Res. 2000;(377):32-43.

26. Moed BR, Yu PH, Gruson KI. Functional outcomes of acetabular fractures. J Bone Joint Surg Am. 2003;85-A(10):1879-83.

27. Rommens PM, Giménez MV, Hessmann MH. Is the posterior wall avulsion the simplest acetabular fracture? Eur J Trauma. 2000;26(4):14454.

28. Routt ML Jr, Simonian PT, Ballmer F. A rational approach to pelvic trauma. Resuscitation and early definitive stabilization. Clin Orthop Relat Res. 1995;(318):61-74.

29. Borrelli J Jr, Koval KJ, Helfet DL. The crescent fracture: a posterior fracture dislocation of the sacroiliac joint. J Orthop Trauma. 1996;10(3): 165-70. 